\section{DIFFERENTIAL CALCULUS WITH SEVERAL VARIABLES}

Vorlesungen über Differential- und Integral-
rechnung
Von Prof. A. Ostrowski. Band 2: Differential-
rechnung auf dem Gebiete mehrerer Variablen.
(Lehrbücher und Monographien aus dem Gebiete der
exakten Wissenschaften : Mathematische Reihe,
Band 5.) Pp. 482. (Basel: Verlag Birkhäuser, 1951.) 67 Swiss francs.

$\mathrm{T}$ HIS is the second volume of Prof. Ostrowski's lectures on differential and integral calculus, intended for independent students as well as for those taking a course at a university. The first volume, dealing with functions of one variable, appeared in 1945 and was reviewed in Nature, 158, 771 (1946). The present volume is described as dealing with the differential calculus in the domain of several variables, but much of it concerns only a single variable. There is to be a third volume, dealing with the more advanced parts of integral calculus.

In the preface, stress is laid on the importance of working examples, of which the book contains a very large number. Some of these are such as appear in other treatises; but more than half of them are original, including some hitherto unpublished theorems. This emphasis on examples is unusual in a Continental book; their value would be even greater if answers were given.

We shall now examine the contents in detail. The first chapter deals with sets of one dimension and of $n$-dimensions. A short second chapter deals with the convergence and continuity of functions of sets. Then a long third chapter (100 pages) gives the most important theorems on infinite sequences and series, and their application to the binomial, exponential, sine and cosine series. Good as this chapter is, it is certainly not concerned with functions of 'seversl' variables, and it might more suitably have appeared in the first volume. The same remark applies also to part of the fourth chapter, on supplements to the differential calculus. This opens with quite elementary theorems on indeterminate forms with infinite numerators and denominators, maxima and minima of functions of a single variable when the second derivative vanishes, and even Leibniz's theorem on the $n$th derivative of a product. It then proceeds to functions of several variables, taking the fundamental theorem to be that of the mean value. It is unfortunate that it is necessary to rely on a formula concerning functions of two variables that was included in the first volume. However, after this, all is clear, and we get an account of partial derivatives, Taylor's theorem for functions of several variables, Euler's theorem on homogeneous functions, the total differential, uniform differentiability, and conditions for the inversion of order of partial differentiation.

The fifth chapter, on applications of the differential calculus to analysis, is one of the best in the book, and deals principally with Jacobians and their application to functional dependence. The theorems are enunciated and proved with unusual care. This chapter also includes an account of implicit functions and of maxima and minima of functions of several variables. The sixth chapter deals with numerical methods, including those of Lagrange, GregoryNewton, Simpson, Gauss, and Newton-Raphson.
Again we remark that the functions concerned are those of a single variable.

The two remaining chapters deal with differential geometry. The seventh chapter starts with the parametric representation of curves in two and three dimensions, and their tangents and normals. Some of this work is surprisingly elementary ; for example, it is explained at length how to find the equation of the tangent at a point of an ellipse, and how to deal with the particular case of the circle. After a brief discussion of singular points it proceeds to rectification and to curvature, evolutes and envelopes of plane curves.

The eighth chapter starts with vectors and their scalar and vector products. It then discusses the osculating plane of a space-curve, and its curvature and torsion, using the moving trihedral and obtaining Frenet's formulæ. The rest of the chapter deals with the properties of surfaces, including tangent planes and normals, and the envelopes of families of surfaces depending on one or two parameters.

The general impression made by the book is very good. The style is clear, and the printing, paper and binding are of very high quality. Unfortunately, the price also is very high. There is no index, but an index to the complete work will appear at the end of the third volume. The reader is warned in the preface that in the second volume 'Sin' and 'Cos' are used for the hyperbolic functions denoted in the first volume by 'sinh' and 'cosh'.

H. T. H. Piaggio

\section{VIRUSES AND MAN}

\section{The Essentials of Virus Diseases}

By Dr. Patrick N. Meenan. Pp. vii + 260. (London : J. and A. Churchill, Ltd., 1951.) 20s. net.

$\mathrm{M}$ ANY people, even teachers of bacteriology, feel that viruses are the subject of an esaterio science, incapable of study or comprehension by ordinary mortals. . As a result, students are often not well instructed about them. Viruses have always been important as causes of disease in man: now that bacterial infections are being better and better controlled by antibiotics, the relative importance of irus diseases becomes steadily greater. Dr. Patrick Meenan, of Dublin, has written a little book in which the "essentials of virus diseases" are presented for the benefit of the practising physician and the medical student; and, be it noted, virus diseases for him are the virus diseases of mankind. He has done a remarkably good job. There are three introductory chapters on characteristics of viruses, methods of study and control; then he gets down to dealing seriatim and in orderly sequence with the essentials of the chief viruses affecting man, in fifteen chapters; there is a final chapter on the collection of specimens for laboratory diagnosis. Viruses which are omitted from his presentation are those of molluscum contagiosum, infectious warts and, surprisingly enough, the common cold.

Though the presentation is in text-book manner, Dr. Meenan has a pleasant, readable style, and his judgment is well balanced. The printers and publishers must have put his material through quickly, for the book is remarkably well up to date, an important point in 'such a rapidly growing subject. If anything, Dr. Meenan is a little too eager to 